\title{
Isothermalisation for a non-local heat equation
}

\author{
EMmanuel Chasseigne AND RAÚl FERREIRA
}

\begin{abstract}
In this paper we study the asymptotic behavior for a nonlocal heat equation in an inhomogenous medium:

$$
\rho(x) u_{t}=J * u-u \text { in } \mathbb{R}^{N} \times(0, \infty),
$$

where $\rho$ is a continuous positive function, $u$ is non-negative and $J$ is a probability measure having finite second-order momentum. Depending on integrability conditions on the initial data $u_{0}$ and $\rho$, we prove various isothermalisation results, i.e., $u(t)$ converges to a constant state in the whole space.
\end{abstract}

Mathematics Subject Classification (2010): 35B40 (primary); 35R09, 45A05, 45K05 (secondary).

\section{Introduction}

The aim of this paper is to study the asymptotic behavior for a nonlocal heat equation in an inhomogenous medium:

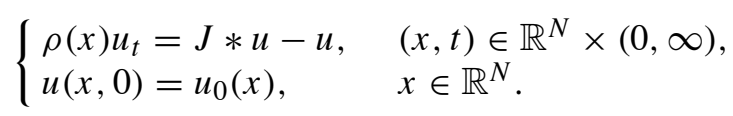

Here, $u_{0}$ is a non-negative continuous function in $\mathbb{R}^{N}$ and $*$ denotes the convolution with a kernel $J: \mathbb{R}^{N} \rightarrow \mathbb{R}$, which is a radial, continuous probability density having finite second-order momentum:

$$
\int_{\mathbb{R}^{N}} J(s) \mathrm{d} s=1, \quad \mathbb{E}(J)=\int_{\mathbb{R}^{N}} s J(s) \mathrm{d} s=0, \mathbb{V}(J)=\int_{\mathbb{R}^{N}} s^{2} J(s) \mathrm{d} s<+\infty .
$$

Typical examples of kernel that we consider are the gaussian law, the exponential law or any compactly supported kernels. We also assume that $\rho$ is a positive, continuous function in $\mathbb{R}^{N}$, whether integrable or not.

Both authors are partially supported by project MTM2008-06326-C02-02 (Spain). R. Ferreira is also partially supported by grant GR58/08-Grupo 920894.

Received December 1, 2011; accepted in revised form October 15, 2012. 
The operator $J * u-u$ can be interpreted as a non-local diffusion operator. Indeed, if $u(x, t)$ represents the density of a single population and $J(x-y)$ is the probability to jump from $y$ to $x$ then the term $(J * u)(x)$ is the rate at which individuals arrive to $x$ and $-u(x)$ is the rate at which individuals leave from $x$, see for instance [6]. In the case of heat propagation, $u$ stands for a temperature and $\rho(x)$ represents the density of the medium.

Problem (1.1) is called non-local because the diffusion at $u(x, t)$ depends on all the values of $u$ in the support of $J$ and not only of the value of $u(x, t)$, as it is the case for the local diffusion problem

$$
\begin{cases}\rho(x) u_{t}=\Delta u, & (x, t) \in \mathbb{R}^{N} \times(0, \infty), \\ u(x, 0)=u_{0}(x), & x \in \mathbb{R}^{N} .\end{cases}
$$

For this local problem it is well known that for dimension $N=1,2$ there exists a unique solution in the class of bounded solutions, see [7] and [4]. Moreover, if $\rho \in \mathrm{L}^{1}\left(\mathbb{R}^{N}\right)$ and $u_{0}$ is bounded, then as $t \rightarrow \infty$ the solution converges on compact sets to $\mathbb{E}_{\rho}\left(u_{0}\right)$, the mean of $u_{0}$ with respect to $\rho$ :

$$
\mathbb{E}_{\rho}\left(u_{0}\right):=\frac{\int_{\mathbb{R}^{N}} u_{0}(y) \rho(y) \mathrm{d} y}{\int_{\mathbb{R}^{N}} \rho(y) \mathrm{d} y} \in \mathbb{R}_{+} .
$$

This phenomenon is called isothermalisation, since the heat distribution converges to a non-trivial isothermal state in all the space. However, for dimension $N \geqslant 3$ uniqueness is lost in the class of bounded solutions and some solutions decrease to zero as $t \rightarrow \infty$, so that isothermalisation does not take place, see [8] and [5].

We are also facing the influence of the space dimension in the nonlocal case. More precisely, if $J$ is compactly supported and $N=1,2$, we prove uniqueness of bounded solutions. For dimension $N \geqslant 3$, we need (as in the local case) additional conditions on the behavior of $\rho$ at infinity to get uniqueness of bounded solutions.

The case when $\rho$ is not integrable is also considered, which is more related to the study of the homogeneous case ( $\rho \equiv 1)$, see [3] and [1]. For bounded solutions, the flux at infinity is so big that solutions go down to zero asymptotically while if the data is unbounded, the solution may go to infinity asymptotically as $t \rightarrow \infty$.

\section{Organization and main results}

We first prove in Section 2 a comparison result which implies uniqueness for problem (1.1) in some class. This comparison states that if $\bar{u}$ is a supersolution of the equation, and $\underline{u}$ is a subsolution, (and both are ordered initially), then we have $\bar{u} \geqslant \underline{u}$ in $\mathbb{R}^{N} \times[0, \infty)$. Such a result is obtained by standard arguments once we are able to construct a suitable strict supersolution (see Lemma 2.5 and Lemma 2.7). But this requires some assumptions on $\rho$. If $\rho$ degenerates too fast at infinity, the maximum principle may be violated. 
In Section 3 we study the existence in the class of bounded solutions, which is obtained by approximation with Neuman problems in bounded domains. Our first main result is the following:

Theorem 1.1. Let $\rho>0$, be continuous, and $u_{0}$ be a bounded non-negative continuous function. Then there exists a non-negative classical bounded solution of problem (1.1). Moreover, if either

$$
\rho(x) \geqslant \frac{\eta}{1+|x|^{2}}
$$

or $N=1,2$ and $J$ has compact support then uniqueness holds in this class.

We denote by $\mathrm{L}^{1}(\rho)$ the space of measurable functions $f: \mathbb{R}^{N} \rightarrow \mathbb{R}$ such that $\rho f \in \mathrm{L}^{1}\left(\mathbb{R}^{N}\right)$. Section 4 is devoted to study the isothermalisation phenomenon for bounded initial data in this space. There are two major tools we use to derive the isothermalisation effect for $u_{0} \in \mathrm{L}^{1}(\rho)$ : (i) an estimate of the solution in $\mathrm{L}^{1}(\rho)$; (ii) some weighted energy estimates in $\mathrm{L}^{2}\left(\mathbb{R}^{N}\right)$. Then the main theorem is as follows:

Theorem 1.2. Let $\rho>0$, be continuous, integrable and $u_{0}$ be a bounded nonnegative continuous function. Then, the solution satisfies the following mass conservation

$$
\int_{\mathbb{R}^{N}} \rho(x) u(x, t) \mathrm{d} x=\int_{\mathbb{R}^{N}} \rho(x) u_{0}(x) \mathrm{d} x .
$$

Moreover, $u(x, t) \rightarrow \mathbb{E}_{\rho}\left(u_{0}\right)$ as $t \rightarrow \infty$ in $\mathrm{L}_{\mathrm{loc}}^{p}\left(\mathbb{R}^{N}\right)$ for any $1 \leqslant p<\infty$; the convergence also holds in $\mathrm{L}^{1}(\rho)$ :

$$
\lim _{t \rightarrow \infty} \int_{\mathbb{R}^{N}}\left|u(x, t)-\mathbb{E}_{\rho}\left(u_{0}\right)\right| \rho(x) \mathrm{d} x=0 .
$$

Notice that since $\rho \in \mathrm{L}^{1}\left(\mathbb{R}^{N}\right)$ and $u_{0}$ is bounded then $u_{0} \in \mathrm{L}^{1}(\rho)$. If $\rho$ is not integrable but still $u_{0} \in \mathrm{L}^{1}(\rho)$ the flux at infinity forces the solution to go to zero:

Theorem 1.3. Let $\rho>0$ and $u_{0}$ be a bounded non-negative continuous function such that $u_{0} \in \mathrm{L}^{1}(\rho)$. If $\rho$ is not integrable in $\mathbb{R}^{N}$, then $u(x, t) \rightarrow 0$ as $t \rightarrow \infty$ in $\mathrm{L}_{\mathrm{loc}}^{p}\left(\mathbb{R}^{N}\right)$ for any $1 \leqslant p<\infty$.

Finally, in Section 5 we investigate the case of unbounded initial data. We first prove an existence result in the class of unbounded solutions provided $\rho$ does not degenerate too rapidly at infinity. More precisely, if

$$
\rho(x) \geqslant \frac{\eta}{1+|x|^{\gamma}} \quad \gamma \leqslant 2
$$

then we have an existence result for quadratic initial data:

Theorem 1.4. Let $u_{0}$ be a positive continuous function with at most quadratic growth at infinity. If the function $\rho$ satisfies (1.3) then there exists a solution of problem (1.1). 
More generally, if $\rho$ is integrable, we prove similar isothermalisation results for the minimal solution:

Theorem 1.5. We assume that $\rho$ is a continuous positive integrable function in $\mathbb{R}^{N}$ and that $u_{0}$ is a continuous non-negative function, possibly unbounded, such that there exists a solution $u$. Noting $\underline{u}$ the minimal solution, the following holds:

i) If $u_{0} \in \mathrm{L}^{1}(\rho)$ the isothermalisation takes place in $\mathrm{L}^{1}(\rho)$,

$$
\lim _{t \rightarrow \infty} \int_{\mathbb{R}^{N}}\left|\underline{u}(x, t)-\mathbb{E}_{\rho}\left(u_{0}\right)\right| \rho(x) \mathrm{d} x=0 .
$$

ii) If $u_{0} \notin \mathrm{L}^{1}(\rho)$, we have that for all $1 \leqslant p<\infty$,

$$
\lim _{t \rightarrow \infty} \underline{u}(x, t)=\infty \quad \text { in } \quad \mathrm{L}_{\mathrm{loc}}^{p}\left(\mathbb{R}^{N}\right) .
$$

In the case of nonintegrable $\rho$ 's with $u_{0} \notin \mathrm{L}^{1}(\rho)$ the asymptotic behavior is more difficult to treat. For instance, if $\rho \equiv 1$, the solutions

$$
u(x, t)=|x|^{2}+\mathbb{V}(J) t, \quad \text { and } \quad u(x, t)=1
$$

have different behavior. Thus, there is a balance between $\rho, J$, and the initial data $u_{0}$ which is not easy to handle and the question remains open.

ACKNOWLEDGEMENTs. We would like to thank Jorge Garcia-Melián for giving us a proof that $J$-harmonic bounded functions are constant (which was reproduced in Lemma 4.4).

\section{Preliminaries}

Let us specify first what is the notion of solution that we use:

Definition 2.1. Let $u_{0} \in \mathrm{L}_{\mathrm{loc}}^{1}\left(\mathbb{R}^{N}\right)$. By a strong solution of (1.1) we mean a function $u \in \mathrm{C}^{0}\left([0, \infty) ; \mathrm{L}_{\mathrm{loc}}^{1}\left(\mathbb{R}^{N}\right)\right)$ such that $u_{t}, J * u \in \mathrm{L}_{\mathrm{loc}}^{1}\left(\mathbb{R}^{N} \times(0, \infty)\right)$, the equation is satisfied in the $\mathrm{L}_{\text {loc }}^{1}$-sense and such that $u(x, 0)=u_{0}(x)$ almost everywhere in $\mathbb{R}^{N}$.

We shall consider also solutions with more regularity:

Definition 2.2. A classical solution of (1.1) is a strong solution such that moreover $u, u_{t}, J * u \in \mathrm{C}^{0}\left(\mathbb{R}^{N} \times[0, \infty)\right)$ and the equation holds in the classical sense everywhere in $\mathbb{R}^{N} \times[0, \infty)$.

A classical sub or supersolution is defined as usual with inequalities instead of equalities in the equation. 
Let us recall some basic facts for (1.1) with $\rho \equiv 1$ (which are more or less valid for a suitable class of functions $\rho$ as we shall see below):

maximum principle If $u$ reaches a max at $x_{0}$, then the convolution $J * u-u$ is necesarily non-negative at $x_{0}$ (this is a consequence of the fact that $J$ is a propability density). And moreover, if $(J * u-u)\left(x_{0}\right)=0$, then $u(x)=u\left(x_{0}\right)$ in the support of $J$, translated at $x_{0}$.

comparison result This kind of nonlocal equation enjoys various comparison principles between sub and supersolutions (whether in $\mathbb{R}^{N}$ of in bounded domains), which rely on the maximum principle above.

existence and uniqueness Both are typically obtained through fixed-point theorems, but uniqueness is also (of course) a consequence of the comparison between sub/supersolutions.

We refer to [1-3] for more details and references.

Now let us state a simple regularity Lemma, which contains a technical trick that we shall use several times in the sequel:

Lemma 2.3. Let $u$ be a strong solution of (1.1). We assume moreover that $u_{0}$ is continuous in $\mathbb{R}^{N}$ and that the convolution term $J * u$ is continuous in $\mathbb{R}^{N} \times[0, \infty)$. Then $u$ and $u_{t}$ are also continuous in $\mathbb{R}^{N} \times[0, \infty)$ and $u$ is a classical solution.

Proof. We introduce the following transform:

$$
\mathcal{T}_{\rho}[u](x, t):=\mathrm{e}^{t / \rho(x)} u(x, t) .
$$

A straightforward calculus show that $v=\mathcal{T}_{\rho}[u]$ satisfies

$$
v_{t}=\frac{\mathrm{e}^{t / \rho(x)}}{\rho(x)}(J * u)(x, t),
$$

which is a continuous function in $\mathbb{R}^{N} \times[0, \infty)$. Integrating between 0 and $t$ we get:

$$
v(x, t)=\int_{0}^{t} \partial_{t} v(x, s) \mathrm{d} s+v(x, 0)=\int_{0}^{t} \partial_{t} v(x, s) \mathrm{d} s+u(x, 0),
$$

hence $v$ is continuous in $\mathbb{R}^{N} \times[0, \infty)$. This implies that $u$ is also continuous in $\mathbb{R}^{N} \times[0, \infty)$, and the equation holds in the classical sense.

Remark 2.4. It is well-known in convolution theory that under one of the following assumptions, the convolution term is continuous:

(i) $u$ bounded (since $J$ is integrable);

(ii) $J$ compactly supported and $u$ locally integrable. 
The following lemma concerns the comparison of classical sub/supersolutions of the problem. In order to prove that we need to find a strict supersolution $\psi$, which satisfies

$$
\begin{aligned}
& \psi \in \mathrm{C}^{0}\left(\mathbb{R}^{N} \times[0, \infty)\right), \psi \geqslant 0, \\
& \psi(x, t) \rightarrow+\infty \text { as }|x| \rightarrow \infty \text { uniformly for } t \in[0, \infty), \\
& \rho \psi_{t}>J * \psi-\psi .
\end{aligned}
$$

Lemma 2.5. In each of the following three cases, if $A>0$ and $\lambda$ is large enough, the function $\psi$ satisfies (2.2):

(i) If function $\rho$ satisfies

$$
\rho(x) \geqslant \frac{\eta}{1+|x|^{2}},
$$

then

$$
\psi(x, t)=A e^{\lambda t}\left(1+|x|^{2}\right) .
$$

(ii) If $J$ has compact support and $N=1$,

$$
\psi(x, t)=A e^{\lambda t}(1+|x|) .
$$

(iii) If $J$ has compact support and $N=2$,

$$
\psi(x, t)=A e^{\lambda t}\left(1+(\ln |x|)_{+}\right) .
$$

Proof. The regularity and the behaviour at infinity of $\psi$ is clear, so we only need to prove that $\psi$ is a strictly supersolution.

In the first case a direct computation gives us that

$$
J * \psi-\psi=A e^{\lambda t} \mathbb{V}(J) .
$$

On the other hand,

$$
\rho \psi_{t}=\rho A \lambda e^{\lambda t}\left(1+|x|^{2}\right) \geqslant A \lambda \eta e^{\lambda t} .
$$

Then, for $\lambda>\mathbb{V}(J) / \eta$, we are done.

In the other two cases, we notice first that $\psi$ is harmonic outside the origin. Since $J$ is a radial probability density supported in $B(0, R)$ (then $\mathbb{E}(J)=0)$, this implies that

$$
J * \psi-\psi=0, \quad \text { for } \quad|x| \geqslant 2 R .
$$

On the other hand, since $\psi$ is continuous in $B(0,2 R)$, there exists $K>0$ such that

$$
J * \psi-\psi \leqslant K e^{\lambda t} \quad \text { for } \quad|x|<2 R .
$$

Moreover, if $|x| \leqslant 2 R$ the function $\rho$ is bounded from below, so there exists a constant $\tilde{K}$ such that

$$
\rho \psi_{t}=\rho \lambda \psi \geqslant \tilde{K} \lambda e^{\lambda t} .
$$

Therefore, taking $\lambda \geqslant K / \tilde{K}$, we get that $\psi$ is a strictly supersolution. 
Remark 2.6. In dimension $N \geqslant 3$ and $J$ compactly supported, the function $\psi(x, t)=e^{\lambda t} \min \left(1,|x|^{2-N}\right)$ would give a strict supersolution, however it goes to zero as $|x| \rightarrow \infty$. Hence it cannot be used to obtain a comparison principle for bounded solutions.

Lemma 2.7. Let $\psi$ satisfy (2.2). Let $\underline{u}$ be a classical subsolution of (1.1) and $\bar{u} a$ classical supersolution of (1.1) such that $\underline{u}(x, 0) \leqslant \bar{u}(x, 0)$. If for all $t \geqslant 0$,

$$
\limsup _{|x| \rightarrow \infty} \frac{u-\bar{u}}{\psi} \leqslant 0
$$

then $\bar{u} \geqslant \underline{u}$ in $\mathbb{R}^{N} \times \mathbb{R}_{+}$.

Proof. We consider the function

$$
w_{\delta}=\underline{u}-\bar{u}-\delta \psi
$$

which satisfies the inequality

$$
\rho(x)\left(w_{\delta}\right)_{t}(x, t)<\left(J * w_{\delta}\right)(x, t)-w_{\delta}(x, t) .
$$

From (2.3), we deduce that on $\mathbb{R}^{N} \times[0, T], w_{\delta}$ attains its maximum at a point $\left(x_{0}, t_{0}\right)$. Observe that at this point,

$$
\rho\left(x_{0}\right)\left(w_{\delta}\right)_{t}\left(x_{0}, t_{0}\right)<\left(J * w_{\delta}\right)\left(x_{0}, t_{0}\right)-w_{\delta}\left(x_{0}, t_{0}\right) \leqslant 0
$$

but if $t_{0} \in(0, T), \partial_{t} w_{\delta}\left(x_{0}, t_{0}\right)=0$ and if $t_{0}=T, \partial_{t} w_{\delta}\left(x_{0}, t_{0}\right) \geqslant 0$. Hence in each of these cases we reach a contradiction. We are left with the last possibility, $t_{0}=0$, which implies $\max w_{\delta} \leqslant 0$. Passing to the limit as $\delta \rightarrow 0$, since $T>0$ is arbitrary we obtain the comparison

$$
\underline{u}-\bar{u} \leqslant 0 \text { for all } x \in \mathbb{R}^{N}, t \geqslant 0 .
$$

As direct corollary, we have the following uniqueness result, which is valid only in a suitable class of solutions, as it is the case for the local heat equation.

Corollary 2.8. Let $\psi$ satisfy (2.2). Let $u_{0}$ be a continuous function which grows strictly less than $\psi$. Then there exists at most one classical solution $u$ of (1.1) such that $|u|$ grows strictly less than $\psi$.

\section{Existence and uniqueness of bounded solutions}

We obtain an existence result by approximation. We consider the following Neumann problem, where $\chi_{n}$ denotes the indicator of the ball $B_{n}$ :

$$
\left\{\begin{array}{l}
\rho(x) \partial_{t} u_{n}=\int_{B_{n}}\left(u_{n}(y, t)-u_{n}(x, t)\right) J(x-y) \mathrm{d} y, \quad x \in B_{n}, \\
u_{n}(x, 0)=u_{0} \chi_{n}(x)
\end{array}\right.
$$

First, let us mention that this problem also enjoys a comparison result: 
Lemma 3.1. Let $\bar{u} \in \mathrm{C}^{1}\left([0, \infty) ; \mathrm{C}^{0}\left(\overline{B_{n}}\right)\right)$ and $\underline{u} \in \mathrm{C}^{1}\left([0, \infty) ; \mathrm{C}^{0}\left(\overline{B_{n}}\right)\right)$ be a supersolution and a subsolution of (3.1) such that $\bar{u}(x, 0) \geqslant \underline{u}(x, 0)$ in $\overline{B_{n}}$. Then $\bar{u} \geqslant \underline{u}$ in $\overline{B_{n}} \times[0, \infty)$.

Proof. The strategy is standard, and follows Lemma 2.7 except that we are in a bounded domain so we need not worry about localizing the supremum with a function $\psi$.

Take $c, T>0$ and consider

$$
w_{c, T}(x, t):=\underline{u}(x, t)-\bar{u}(x, t)-\frac{c}{T-t}
$$

in $\overline{B_{n}} \times[0, T]$. This function satisfies the inequality

$$
\rho(x) \partial_{t} w_{c, T} \leqslant \int_{B_{n}}\left(w_{c, T}(y, t)-w_{c, T}(x, t)\right) J(x-y) \mathrm{d} y-\frac{c \rho(x)}{(T-t)^{2}} .
$$

Notice that this inequality is still valid if $x_{0} \in \partial B_{n}$, this is just using the Dominated Convergence Theorem with a sequence $x_{n} \rightarrow x_{0}, x_{n} \in B_{n}$.

We shall prove that for any $c, T>0$, we have $\max w_{c, T} \leqslant 0$. Of course $w_{c, T}$ reaches its maximum in $\bar{B}_{n} \times[0, T]$ because it is continuous, and obviously it cannot be attained at $t=T$. If this max was attained at $t=0$, we would deduce that $\max w_{c, T} \leqslant 0$ which is what we want.

So, let us assume that the maximum point $\left(x_{0}, t_{0}\right)$ is such that $0<t<T$. Hence, by the above inequality we get the following contradiction

$$
\begin{aligned}
0 & =\rho\left(x_{0}\right) \partial_{t} w_{c, T}\left(x_{0}, t_{0}\right) \\
& \leqslant \int_{B_{n}}\left(w_{c, T}\left(y, t_{0}\right)-w_{c, T}\left(x_{0}, t_{0}\right)\right) J\left(x_{0}-y\right) \mathrm{d} y-\frac{c \rho\left(x_{0}\right)}{\left(T-t_{0}\right)^{2}}<0 .
\end{aligned}
$$

So we are left with $t_{0}=0$ and $\max w_{c, T} \leqslant 0$.

Passing to the limit as $c \rightarrow 0$ we deduce that $\underline{u} \leqslant \bar{u}$ in $B_{n} \times[0, T]$, and then we send $T \rightarrow \infty$ to finish the proof.

Lemma 3.2. Let $\rho>0$ be a continuous function in $\mathbb{R}^{N}$ and $u_{0}$ be a non-negative bounded continuous function. Then for any $n \geqslant 0$, there exists a unique solution $u_{n} \in \mathrm{C}^{1}\left([0, \infty) ; \mathrm{C}^{0}\left(\overline{B_{n}}\right)\right)$, which satisfies $0 \leqslant u_{n} \leqslant\left\|u_{0}\right\|_{\infty}$. Moreover, the following conservation law holds:

$$
\int_{B_{n}} u_{n}(x, t) \rho(x) \mathrm{d} x=\int_{B_{n}} u_{0}(x) \rho(x) \mathrm{d} x .
$$

Proof. Following [3], we consider $t_{0}>0$ to be fixed later on and the Banach space $X_{t_{0}}=\mathrm{C}^{0}\left(\left[0, t_{0}\right] \times \overline{B_{n}}\right)$ equipped with the norm $\|w\|=\max \left\{\|w(\cdot, t)\|_{\mathrm{L}^{\infty}\left(\overline{B_{n}}\right)}, 0 \leqslant\right.$ $\left.t \leqslant t_{0}\right\}$. Then we define an operator $T: X_{t_{0}} \rightarrow X_{t_{0}}$ as follows:

$$
T_{w_{0}}(w)(x, t):=w_{0}(x)+\frac{1}{\rho} \int_{0}^{t} \int_{B_{n}} J(x-y)(w(y, s)-w(x, s)) \mathrm{d} y \mathrm{~d} s .
$$


A straightforward calculus, using that $\rho \geqslant \rho_{0}$ in $B_{n}$ shows that:

$$
\left\|T_{w_{0}}(w)-T_{z_{0}}(z)\right\| \leqslant\left\|w_{0}-z_{0}\right\|_{L^{\infty}\left(\overline{B_{n}}\right)}+\frac{2 t_{0}}{\rho_{0}}\|w-z\| .
$$

We deduce that for any fixed initial data $u_{0} \in \mathrm{L}^{\infty}\left(\overline{B_{n}}\right)$, if $t_{0}>0$ is sufficiently small (say $t_{0}<\rho_{0} / 4$ ), $T_{u_{0}}$ is a strict contraction in the Banach space $X_{t_{0}}$. Hence there exists a unique function $u_{n} \in X_{t_{0}}$ such that $T_{u_{0}}\left(u_{n}\right)=u_{n}$, which means that for any $x \in \overline{B_{n}}$ and $t \in\left[0, t_{0}\right]$, one has

$$
u_{n}(x, t)=u_{0}(x)+\frac{1}{\rho} \int_{0}^{t} \int_{B_{n}} J(x-y)\left(u_{n}(y, s)-u_{n}(x, s)\right) \mathrm{d} y \mathrm{~d} s .
$$

This in particular implies that $u(0)=u_{0}$ and moreover, that the weak derivative $\partial_{t} u$ exists, which is given by

$$
\partial_{t} u_{n}=\frac{1}{\rho} \int_{B_{n}} J(x-y)\left(u_{n}(y, s)-u_{n}(x, s)\right) \mathrm{d} y .
$$

Since the right hand side of the equation is a continuous function in $\overline{B_{n}} \times\left[0, t_{0}\right]$, we have that $\partial_{t} u$ is also continuous in $\overline{B_{n}} \times\left[0, t_{0}\right]$. To extend the solution to $[0, \infty)$ we take as initial data $u\left(x, t_{0}\right) \in C^{0}\left(\overline{B_{n}}\right)$ and obtain a solution up [0,2to]. We then iterate the procedure to construct a solution for all time $t>0$.

On the other hand, we note that the constants functions $\bar{u}=\left\|u_{0}\right\|_{\infty}$ and $\underline{u}=0$ are a supersolution and subsolution respectively, then by comparison

$$
0 \leqslant u_{n} \leqslant\left\|u_{0}\right\|_{\infty}
$$

Finally, we observe that

$$
\rho(x) u_{n}(x, t)-\rho(x) u_{0}(x)=\int_{0}^{t} \int_{B_{n}} J(x-y)\left(u_{n}(y, s)-u_{n}(x, s)\right) \mathrm{d} y \mathrm{~d} s .
$$

Then, integrating in $x$ and apply Fubini's theorem in the right hand side of the equation, we get de desired conservation law.

Proposition 3.3. Let $\rho>0$ be a continuous function in $\mathbb{R}^{N}$ and $u_{0}$ be a nonnegative continuous bounded function and for any integer $n>0$, let $u_{n}$ be the solution constructed in Lemma 3.2. Then as $n \rightarrow \infty$, along a subsequence $u_{n} \rightarrow u$ in $\mathrm{L}_{\text {loc }}^{1}\left(\mathbb{R}^{N} \times[0, \infty)\right.$ ) where $u$ is a non-negative classical bounded solution of (1.1).

Proof. Since the sequence $u_{n}$ is bounded, it converges (along a subsequence still denoted $u_{n}$ ) in $\mathrm{L}^{\infty}$-weak* to some non-negative and bounded function $u$. Since $J \in \mathrm{L}^{1}$, this implies that $J * u_{n} \rightarrow J * u$ strongly and that $\partial_{t} u_{n} \rightarrow \partial_{t} u$ in the sense of distributions. We can then pass to the limit in the sense of distributions but here also we want a better convergence. 
Let us introduce a modified version of transform $\mathcal{T}$ as follows:

$$
v_{n}(x, t):=\mathrm{e}^{t\left(J * \chi_{n}\right)(x) / \rho(x)} u_{n}(x, t) .
$$

Since (3.1) can be written as

$$
\rho(x) \partial_{t}\left(u_{n}\right)(x, t)=\left[J *\left(u_{n} \chi_{n}\right)\right](x, t)-\left(J * \chi_{n}\right)(x) u_{n}(x),
$$

it follows immediately that $v_{n}$ satisfies the equation

$$
\partial_{t} v_{n}=\frac{\mathrm{e}^{t\left(J * \chi_{n}\right)(x) / \rho(x)}}{\rho(x)} J * u_{n} .
$$

This implies that $\partial_{t} v_{n}$ converges strongly on compact sets of $\mathbb{R}^{N} \times[0, \infty)$, and so does $v_{n}(x, t)=u_{0}(x) \chi_{n}(x)+\int_{0}^{t}\left(v_{n}\right)_{t}(x, s) \mathrm{d} s$. Then $u_{n}$ also converges strongly on compact sets of $\mathbb{R}^{N} \times[0, \infty)$ to its limit $u$.

Passing to the limit in the equation, we see that $u$ is a strong solution of (1.1), which implies that it is a classical solution of this equation - this follows from Lemma 2.3.

Proof of Theorem 1.1. It is just the combination of Proposition 3.3, Corollary 2.8 and Lemma 2.5.

Now, we pass to the limit in the conservation law, to obtain:

Theorem 3.4. Let $\rho>0$, be continuous and $u_{0} \in \mathrm{C}^{0}\left(\mathbb{R}^{N}\right) \cap \mathrm{L}^{\infty}\left(\mathbb{R}^{N}\right) \cap \mathrm{L}^{1}(\rho)$. Then:

(i) for any $t>0, u(\cdot, t) \in \mathrm{L}^{1}(\rho)$ and

$$
\int_{\mathbb{R}^{N}} \rho(x) u(x, t) \mathrm{d} x \leqslant \int_{\mathbb{R}^{N}} \rho(x) u_{0}(x) \mathrm{d} x .
$$

(ii) if moreover we assume that $\rho \in \mathrm{L}^{1}\left(\mathbb{R}^{N}\right)$ then the conservation law holds:

$$
\int_{\mathbb{R}^{N}} \rho(x) u(x, t) \mathrm{d} x=\int_{\mathbb{R}^{N}} \rho(x) u_{0}(x) \mathrm{d} x .
$$

Proof. Since $u_{0} \in \mathrm{L}^{\infty} \cap \mathrm{C}^{0}$, the approximating sequence $\left\{u_{n}\right\}$ of Proposition 3.3 satisfies that (up to a subsequence) $u_{n} \rightarrow u$ almost everywhere, with $0 \leqslant u_{n} \leqslant$ $\left\|u_{0}\right\|$ and

$$
\int_{\mathbb{R}^{N}} u_{n}(x, t) \chi_{n}(x) \rho(x) \mathrm{d} x=\int_{\mathbb{R}^{N}} u_{0}(x) \chi_{n}(x) \rho(x) \mathrm{d} x .
$$

Since $u_{0} \in \mathrm{L}^{1}(\rho)$, the dominated convergence theorem yields the convergence of the right-hand side integral as $n \rightarrow \infty$. For the left hand side, using Fatou's Lemma we obtain that

$$
\int_{\mathbb{R}^{N}} \rho(x) u(x, t) \mathrm{d} x \leqslant \liminf _{n \rightarrow \infty} \int_{\mathbb{R}^{N}} \rho(x) u_{n}(x, t) \chi_{n}(x) \mathrm{d} x=\int_{\mathbb{R}^{N}} \rho(x) u_{0}(x) \mathrm{d} x,
$$

which proves assumption (i). 
Finally, if we assume that $\rho \in \mathrm{L}^{1}\left(\mathbb{R}^{N}\right)$ we can use also the dominated convergence theorem for the sequence $u_{n} \chi_{n} \rho$, which is bounded by $\left\|u_{0}\right\|_{\infty} \rho \in \mathrm{L}^{1}\left(\mathbb{R}^{N}\right)$. We then pass to the limit in the left-hand side of (3.3) and get (ii).

\section{Asymptotic behaviour for bounded solutions}

We shall now derive our main results concerning the asymptotic behaviour for (1.1). We divide the proof in several steps.

\subsection{Weak limit}

This first step is easy, it only comes from the fact that the solution is globally bounded:

Lemma 4.1. Let $\rho>0$, continuous and $u_{0} \in \mathrm{C}^{0}\left(\mathbb{R}^{N}\right) \cap \mathrm{L}^{\infty}\left(\mathbb{R}^{N}\right)$. Let $u$ be a bounded classical solution. Then for any $s>0$ there exists a subsequence $t_{k} \rightarrow$ $+\infty$ such that the following limit exists in $\mathrm{L}^{\infty}$-weak*

$$
u_{\infty}(x, s):=\lim _{t_{k} \rightarrow \infty} u\left(x, s+t_{k}\right)
$$

Proof. Since $u$ is bounded, thus there exists a subsequence $t_{k} \rightarrow \infty$ such that $u\left(\cdot, s+t_{k}\right)$ converges in $\mathrm{L}^{\infty}$-weak* to a function $u_{\infty}(\cdot, s) \in \mathrm{L}^{\infty}\left(\mathbb{R}^{N}\right)$.

\subsection{Lyapounov functional}

We now want a stronger result, so we use a Lyapounov functional:

Lemma 4.2. Let $t_{0}>0$. Assume the hypotheses of Lemma 4.1 and that $u_{0} \in \mathrm{L}^{2}(\rho)$. Then there exists a constant $C=C\left(u_{0}, \rho, t_{0}\right)$ such that for all $t \geqslant t_{0}>0$,

$$
\int_{t}^{\infty} \int_{\mathbb{R}^{N}} \rho(x)\left(u_{t}\right)^{2}(x, s) \mathrm{d} s \leqslant C .
$$

Proof. We first go back to the approximating scheme (3.1). Notice that the following quantity

$$
F\left[u_{n}\right](t)=\int_{B_{n}} \int_{B_{n}} J(x-y)\left(u_{n}(x, t)-u_{n}(y, t)\right)^{2} \mathrm{~d} x \mathrm{~d} y
$$


is a Lyapounov functional which is nonincreasing along the evolution orbits at the level $n$. Indeed, multiplying the equation by $u$ and integrating in $B_{n}$ we get

$$
\begin{aligned}
\int \rho(x) \partial_{t} u_{n} \cdot u_{n} \mathrm{~d} x & =\iint J(x-y) u_{n}(x) u_{n}(y) \mathrm{d} x \mathrm{~d} y-\int u_{n}(x)^{2} \mathrm{~d} x \\
& =\frac{1}{2} \iint J(x-y)\left(2 u_{n}(x) u_{n}(y)-2 u_{n}(x)^{2}\right) \mathrm{d} x \mathrm{~d} y \\
& =\frac{1}{2} \iint J(x-y)\left(2 u_{n}(x) u_{n}(y)-u_{n}(x)^{2}-u_{n}(y)^{2}\right) \mathrm{d} x \mathrm{~d} y \\
& =-\frac{1}{2} F\left[u_{n}\right](t) .
\end{aligned}
$$

Therefore,

$$
F\left[u_{n}\right](t)=-\frac{\mathrm{d}}{\mathrm{d} t} \int_{B_{n}} \rho_{n}(x) u_{n}^{2}(x, t) \mathrm{d} x .
$$

In a similar way, multiplying the equation by $\partial_{t} u_{n}$ and integrating in space we obtain

$$
\frac{\mathrm{d}}{\mathrm{d} t} F\left[u_{n}\right](t)=-4 \int_{B_{n}} \rho(x)\left(\left(u_{n}\right)_{t}\right)^{2}(x, t) \mathrm{d} x .
$$

Integrating (4.1) we have that for some $C^{\prime}=C^{\prime}\left(u_{0}, \rho\right)$,

$$
\int_{0}^{t} F\left[u_{n}\right](s) \mathrm{d} s=2 \int_{B_{n}} \rho(x) u_{0}^{2}(x) \chi_{n}(x) \mathrm{d} x-2 \int_{B_{n}} \rho(x) u_{n}^{2}(x, t) \mathrm{d} x \leqslant C^{\prime} .
$$

Indeed, as $u_{0} \in \mathrm{L}^{2}(\rho)$ we have by monotone convergence that

$$
\int_{\mathbb{R}^{N}} \rho(x) u_{0}^{2}(x) \chi_{n}(x) \mathrm{d} x \rightarrow \int_{\mathbb{R}^{N}} \rho(x) u_{0}^{2}(x) \mathrm{d} x<\infty .
$$

Using now the monotonicity in $t$ of $F\left[u_{n}\right](t)$, we get

$$
t F\left[u_{n}\right](t) \leqslant \int_{0}^{t} F\left[u_{n}\right](s) \mathrm{d} s \leqslant C^{\prime}
$$

Moreover, $F\left[u_{n}\right](t)$ is positive so that, integrating (4.2), we get for any $t \geqslant t_{0}$ :

$$
\int_{t}^{\infty} \int_{\mathbb{R}^{N}} \rho\left(\left(u_{n}\right)_{t}\right)^{2}(x, s) \mathrm{d} x \mathrm{~d} s \leqslant \frac{1}{4} F\left[u_{n}\right](t) \leqslant \frac{C^{\prime}}{4 t_{0}} .
$$

Using Fatou's Lemma and the fact that $\rho\left(u_{n}\right)_{t}$ converges strongly to $\rho u_{t}$, we obtain the desired result. 
As an immediate consequence of this result we obtain:

Lemma 4.3. Assume the hypotheses of Lemma 4.1 and that $u_{0} \in \mathrm{L}^{2}(\rho)$. For all sequence $t_{k} \rightarrow \infty$ and $s>0$,

$$
\left\|\sqrt{\rho(\cdot)} u\left(\cdot, s+t_{k}\right)-\sqrt{\rho(\cdot)} u\left(\cdot, t_{k}\right)\right\|_{\mathrm{L}^{2}\left(\mathbb{R}^{N}\right)}^{2} \rightarrow 0 \quad \text { as } n \rightarrow \infty .
$$

Hence, the limit function $u_{\infty}(x, s)$ does not depend on the variable $s>0$.

Proof. Notice that for all sequence $t_{k} \rightarrow \infty$, we get

$$
\begin{aligned}
\left\|\sqrt{\rho(\cdot)} u\left(\cdot, s+t_{k}\right)-\sqrt{\rho(\cdot)} u\left(\cdot, t_{k}\right)\right\|_{\mathrm{L}^{2}\left(\mathbb{R}^{N}\right)}^{2} & =\int_{\mathbb{R}^{N}} \rho(x)\left(\int_{t_{k}}^{t_{k}+s} u_{t}(x, \sigma) \mathrm{d} \sigma\right)^{2} \mathrm{~d} x \\
& \leqslant s \int_{\mathbb{R}^{N}} \int_{t_{k}}^{t_{k}+s} \rho(x)\left(u_{t}\right)^{2}(x, \sigma) \mathrm{d} \sigma \mathrm{d} x,
\end{aligned}
$$

which goes to zero as $t_{k} \rightarrow+\infty$

\subsection{The $\omega$-limit set}

We define the $\omega$-limit set as follows:

$$
\omega\left(u_{0}\right)=\left\{u_{\infty} \in \mathrm{C}^{0}\left(\mathbb{R}^{N}\right): \exists t_{j} \rightarrow \infty \text { such that } u\left(\cdot, t_{j}\right) \rightarrow u_{\infty}(\cdot) \text { in } \mathrm{L}^{\infty} \text {-weak }{ }^{*}\right\} .
$$

Lemma 4.4. Under the hypotheses of Lemma 4.1 and that $u_{0} \in \mathrm{L}^{2}(\rho)$, the $\omega$-limit set is reduced to constants.

Proof. Since $u\left(x, s+t_{k}\right)$ converges weakly in $\mathrm{L}^{\infty}$-weak*, then as $t_{k} \rightarrow \infty$,

$$
(J * u)\left(x, s+t_{k}\right)=\int J(x-y) u\left(y, s+t_{k}\right) \mathrm{d} y \stackrel{\text { pointwise }}{\longrightarrow}\left(J * u_{\infty}\right)(x, s) .
$$

Moreover, since $u$ is bounded, the convergence of $J * u$ is also strong in $\mathrm{L}_{\text {loc }}^{1}$. On the other hand, $\partial_{t} \rho u\left(s+t_{k}\right) \rightarrow \rho \partial_{s} u_{\infty}(s)$ in the sense of distributions. We then pass to the limit in the sense of distributions in the equation and get

$$
\rho(x) \frac{\partial}{\partial s} u_{\infty}(x, s)=J * u_{\infty}(x, s)-u_{\infty}(x, s) .
$$

Using Lemma 4.3 we know that $u_{\infty}$ is independent of $s$ so that $u_{\infty}$ is a bounded solution (in the sense of distributions) of

$$
J * u_{\infty}-u_{\infty}=0 \text { in } \mathbb{R}^{N} .
$$

We first deduce that $u_{\infty}$ is continuous because the convolution term is continuous. Then we consider the Fourier transform in the space of tempered distributions:

$$
\hat{J} \cdot \hat{u}_{\infty}-\hat{u}_{\infty}=0 .
$$

Since $|\hat{J}|<1$ except for $\hat{J}(0)=1$, we see that the (generalized) Fourier transform of $u_{\infty}$ has a support contained in $\{0\}$. This implies that $u_{\infty}$ is a polynom but since it is bounded, it has to be a constant. 


\subsection{Identification of the limit}

We are now ready to identify the $\omega$-limit set.

Lemma 4.5. We assume the hypotheses of Lemma 4.1 and that $u_{0} \in \mathrm{L}^{1}(\rho)$. Then the following holds:

(i) if $\rho \in \mathrm{L}^{1}\left(\mathbb{R}^{N}\right), \omega\left(u_{0}\right)=\left\{\mathbb{E}_{\rho}\left(u_{0}\right)\right\}$;

(ii) if $\rho \notin \mathrm{L}^{1}\left(\mathbb{R}^{N}\right), \omega\left(u_{0}\right)=\{0\}$.

Proof. Notice first that since $u_{0} \in \mathrm{L}^{1}(\rho) \cap \mathrm{L}^{\infty}$, then $u_{0} \in \mathrm{L}^{2}(\rho)$, hence we may use Lemma 4.4. In the integrable case, $\rho \in \mathrm{L}^{1}$, we observe that as $u$ is uniformly bounded, the dominated convergence Theorem gives

$$
\int_{\mathbb{R}^{N}} \rho(x) u_{n}\left(x, s+t_{j}\right) \mathrm{d} x \rightarrow u_{\infty} \int_{\mathbb{R}^{N}} \rho(x) \mathrm{d} x .
$$

Therefore, by Theorem 3.4-(ii) we obtain $u_{\infty}=\mathbb{E}_{\rho}\left(u_{0}\right)$, so that the $\omega$-limit set is reduced to $\left\{\mathbb{E}_{\rho}\left(u_{0}\right)\right\}$.

In the case $\rho \notin \mathrm{L}^{1}\left(\mathbb{R}^{N}\right)$, we take a compact set $K$ such that

$$
\int_{K} \rho(x) \mathrm{d} x>\int_{\mathbb{R}^{N}} \rho(x) u_{0}(x) \mathrm{d} x,
$$

which is always possible since $u_{0} \in \mathrm{L}^{1}(\rho)$. Using Theorem 3.4-(i), Lemma 4.4 and Fatou's Lemma, we obtain

$$
\begin{aligned}
u_{\infty} \int_{K} \rho(x) \mathrm{d} x & \leqslant \liminf _{n \rightarrow \infty} \int_{K} \rho(x) u_{n}\left(x, s+t_{j}\right) \mathrm{d} x \\
& \leqslant \liminf _{n \rightarrow \infty} \int_{\mathbb{R}^{N}} \rho(x) u_{n}\left(x, s+t_{j}\right) \mathrm{d} x \\
& \leqslant \int_{\mathbb{R}^{N}} \rho(x) u_{0}(x) \mathrm{d} x .
\end{aligned}
$$

This implies that necessarily $u_{\infty}=0$, hence the $\omega$-limit set is reduced to $\{0\}$.

\subsection{Proofs of Theorems 1.2 and 1.3}

As consequence of the fact that the $\omega$-limit is given by only one function we can pass to the limit in the time variable without extracting any subsequence. Then it only remains to check that the convergence is better, which is done by using transform $\mathcal{T}_{\rho}$.

Proof of Theorem 1.3. Under the hypotheses of the Theorem, we have that $u_{0}$ is continuous, bounded, and $\rho$ not integrable, but nevertheless $u_{0} \in \mathrm{L}^{1}(\rho) \cap \mathrm{L}^{2}(\rho)$. Thus Lemmas 4.1 and 4.5 imply that for any $s>0$, at least along a subsequence 
$t_{n} \rightarrow \infty$ we have $u\left(x, s+t_{n}\right) \rightarrow 0$ in $\mathrm{L}^{\infty}$-weak*. But the same arguments are valid for any other subsequence such that $u\left(x, s+t^{\prime}{ }_{n}\right)$ converges weakly. Since the limit is always zero, we deduce that for any $s>0$,

$$
u(x, s+t) \stackrel{\mathrm{L}^{\infty} \text {-weak* }}{\underset{t \rightarrow \infty}{\longrightarrow}} 0,
$$

which implies that $(J * u(s+t))$ converges strongly in $\mathrm{L}_{\text {loc }}^{1}$ as $t \rightarrow \infty$. Then,

$$
\rho(x) \partial_{s} u(x, s+t)=(J * u(s+t))(x)-u(x, s+t) \underset{t \rightarrow \infty}{\stackrel{\mathrm{L}^{\infty} \text {-weak* }}{\longrightarrow}} 0 .
$$

Even more, since $t \rightarrow+\infty$ we may assume that $t>t_{0}$ for some $t_{0}>0$ and from lemma 4.2 we obtain that for any compact set $K$,

$$
\begin{aligned}
\int_{t}^{\infty}\left(\int_{K} \rho(x)\left|u_{t}\right|(x, s) \mathrm{d} x\right)^{2} \mathrm{~d} s & \leqslant \int_{t}^{\infty}\left(\int_{K} \rho(x)\left|u_{t}\right|^{2}(x, s) \mathrm{d} x\right) \\
& \leqslant\left(\int_{K} \rho(x) \mathrm{d} x\right) \mathrm{d} s \leqslant C\left(K, u_{0}, \rho\right) .
\end{aligned}
$$

Then, at least for some sequence $t_{k} \rightarrow+\infty$, we have $\rho(x) \partial_{s} u\left(x, s+t_{k}\right) \rightarrow 0$ in $\mathrm{L}_{\text {loc }}^{1}$. Summing up, we obtain that

$$
\lim _{t_{k} \rightarrow+\infty} u\left(x, s+t_{k}\right)=0 \text { in } \mathrm{L}_{\text {loc }}^{1}
$$

Of course, if $t \mapsto u(x, s+t)$ were to converge in $\mathrm{L}_{\text {loc }}^{1}$ along another subsequence $t_{k}^{\prime} \rightarrow \infty$, the limit would necessarily be zero, so that finally $u(\cdot, t) \rightarrow 0$ in $\mathrm{L}_{\text {loc }}^{1}\left(\mathbb{R}^{N}\right)$ as $t \rightarrow \infty$. Moreover, since $t \mapsto u(\cdot, t)$ remains bounded in $\mathrm{L}^{\infty}\left(\mathbb{R}^{N}\right)$, we deduce that the convergence holds in $\mathrm{L}_{\text {loc }}^{p}\left(\mathbb{R}^{N}\right)$ for any $1 \leqslant p<\infty$.

Proof of Theorem 1.2. The first part is done exactly as in the proof of Theorem 1.3, except that $\rho$ is integrable here so that the limit is not zero, but $\mathbb{E}_{\rho}\left(u_{0}\right)$. To end the proof in this case, it only remains to prove the $\mathrm{L}^{1}(\rho)$ convergence. We fix $\varepsilon>0$ and choose $R>0$ big enough so that (remember that $\rho$ is integrable):

$$
\int_{|x|>R} \rho(x) \mathrm{d} x \leqslant \varepsilon .
$$

Then

$$
\int_{\mathbb{R}^{N}}\left|u(x, t)-u_{\infty}\right| \rho(x) \mathrm{d} x \leqslant 2 \varepsilon\left\|u_{0}\right\|_{\infty}+\int_{|x| \leqslant R}\left|u(x, t)-u_{\infty}\right| \rho(x) \mathrm{d} x,
$$

and using the $\mathrm{L}_{\text {loc }}^{1}$ convergence we get:

$$
\limsup _{t \rightarrow \infty} \int_{\mathbb{R}^{N}}\left|u(x, t)-u_{\infty}\right| \rho(x) \mathrm{d} x \leqslant 2 \varepsilon\left\|u_{0}\right\|_{\infty} .
$$

Since $\varepsilon$ is arbitrary, we get that the limit is zero, which ends the proof. 


\section{Unbounded solutions}

In this section we derive some results for unbounded initial data and solutions. Let us mention that in the case $\rho \equiv 1$, further results are to be found in [1]. But here we still face the problem of the space inhomogeneity implied by $\rho$.

\subsection{An existence result for unbounded solutions}

Proposition 5.1. Let us assume that (2.2) holds. Then for any non-negative $u_{0} \in$ $\mathrm{C}^{0}\left(\mathbb{R}^{N}\right)$, satisfying $u_{0}(x) \leqslant \psi(x, 0)$ there exist a classical solution $u$ of $(1.1)$ with $u(x, 0)=u_{0}(x)$.

Proof. Let us first consider an approximation $u_{0 n}=u_{0} \cdot \chi_{n}$ where $\chi_{n}$ is smooth, non-negative, compactly supported and $\chi_{n} \nearrow 1$. Let $u_{n}$ be the solution of (1.1) with initial data $u_{0 n}$ given by Proposition 3.3, then by applying the comparison result for bounded solutions, the sequence $u_{n}$ is nondecreasing.

Notice that $\psi$ is not bounded, but this is allowed in Lemma 2.7, which gives:

$$
u_{n}(x, t) \leqslant \psi(x, t) .
$$

Hence the sequence $u_{n}$ converges to some $u$ and we are able to pass to the limit in $J * u_{n}$ by dominated convergence, using that $\psi$ is integrable with respect to translations of $J$.

Using now Lemma 2.3, we deduce that $u$ is a classical solution of (1.1) and the initial data of $u$ is $u_{0}$.

Remark 5.2. This construction does in fact give a minimal solution: if $u_{1}$ is any other solution, then it can be used as a supersolution for any $u_{n}$ and passing to the limit shows that $u \leqslant u_{1}$. One can think that if we restrict the initial data to grow strictly less than $\psi$, then uniqueness holds because the comparison argument is valid in this class. However, it is not clear whether the constructed solution enters this class unless we know more about $u_{0}$, see [1].

\subsection{Asymptotic behaviour for unbounded solutions when $\rho$ is integrable}

We prove now that if $u_{0}$ is integrable with respect to $\rho$, the isothermalisation phenomenon occurs (whether infinite or not). Notice that we gave sufficient conditions for existence of a minimal solution in the previous section. The first result is the following:

Proposition 5.3. Let $u_{0} \in \mathrm{C}^{0}\left(\mathbb{R}^{N}\right) \cap \mathrm{L}^{1}(\rho), \rho \in \mathrm{L}^{1}\left(\mathbb{R}^{N}\right)$ and assume there exists a solution $u$ such that $u(x, 0)=u_{0}(x)$. Then, if $\underline{u}$ denotes the minimal solution, we have

$$
\lim _{t \rightarrow \infty} \int_{\mathbb{R}^{N}}\left|\underline{u}(x, t)-\mathbb{E}_{\rho}\left(u_{0}\right)\right| \rho(x) \mathrm{d} x=0 .
$$


Proof. Let $\underline{u}$ be the minimal solution and let us use the same monotone approximations that were used in Proposition 5.1. Since $u_{n}$ is bounded, Lemma 2.7 implies that we have a bound from above:

$$
u(x, t) \geqslant u_{n}(x, t) \text { in } \mathbb{R}^{N} \times[0, \infty) .
$$

But since $u_{n}(x, 0) \in \mathrm{L}^{\infty}\left(\mathbb{R}^{N}\right)$, Theorem 3.4 implies

$$
\int_{\mathbb{R}^{N}} u_{n}(x, t) \rho(x) \mathrm{d} x=\int_{\mathbb{R}^{N}} u_{n}(x, 0) \rho(x) \mathrm{d} x .
$$

Moreover, the convergence of $u_{n}$ to $\underline{u}$ is monotone, so we can pass to the limit in the above equation to obtain

$$
\int_{\mathbb{R}^{N}} \underline{u}(x, t) \rho(x) \mathrm{d} x=\int_{\mathbb{R}^{N}} \underline{u}(x, 0) \rho(x) \mathrm{d} x .
$$

Using the above three equations we get that

$$
\int_{\mathbb{R}^{N}}\left|\underline{u}(x, t)-\mathbb{E}_{\rho}\left(u_{0}\right)\right| \rho(x) \mathrm{d} x \leqslant I_{1}+I_{2}+I_{3}
$$

where,

$$
\begin{gathered}
I_{1}=\int_{\mathbb{R}^{N}}\left|\underline{u}(x, t)-u_{n}(x, t)\right| \rho(x) \mathrm{d} x=\int_{\mathbb{R}^{N}}\left(\underline{u}(x, 0)-u_{n}(x, 0)\right) \rho(x) \mathrm{d} x . \\
I_{2}=\int_{\mathbb{R}^{N}}\left|u_{n}(x, t)-\mathbb{E}_{\rho}\left(u_{0} \chi_{n}\right)\right| \rho(x) \mathrm{d} x, \\
I_{3}=\int_{\mathbb{R}^{N}}\left|\mathbb{E}_{\rho}\left(u_{0} \chi_{n}\right)-\mathbb{E}_{\rho}\left(u_{0}\right)\right| \rho(x) \mathrm{d} x .
\end{gathered}
$$

Observe that $I_{1}$ and $I_{3}$ are independents of $t$ and tend to zero as $n \rightarrow \infty$. Moreover, $u_{n}$ satisfies the hypothesis of Theorem 1.2 so that $I_{2}$ tends to zero as $t \rightarrow \infty$. Therefore, we first have

$$
\limsup _{t \rightarrow+\infty} \int_{\mathbb{R}^{N}}\left|\underline{u}(x, t)-\mathbb{E}_{\rho}\left(u_{0}\right)\right| \rho(x) \mathrm{d} x \leqslant I_{1}+I_{3},
$$

so that taking the limit as $n \rightarrow \infty$ yields the desired result.

In the case when $u_{0} \notin \mathrm{L}^{1}(\rho)$, then infinite isothermalisation occurs:

Proposition 5.4. Let $\rho \in \mathrm{L}^{1}\left(\mathbb{R}^{N}\right)$ and $u_{0} \in \mathrm{C}^{0}\left(\mathbb{R}^{N}\right)$ be such that $u_{0} \notin \mathrm{L}^{1}(\rho)$. Then for any solution $u$ with initial data $u_{0}$ and any $1 \leqslant p<\infty$, the following asymptotic behaviour holds:

$$
\lim _{t \rightarrow+\infty} u(x, t)=+\infty \text { in } \mathrm{L}_{\mathrm{loc}}^{p}\left(\mathbb{R}^{N}\right) .
$$


Proof. As before, if there exists a solution, then we can approximate the minimal solution $\underline{u}$ by the family $u_{n}$ used in Proposition 5.1. Since this approximation is monotone,

$$
\underline{u}(x, t) \geqslant u_{n}(x, t) \text { in } \mathbb{R}^{N} \times[0, \infty) .
$$

But $u_{n}(x, 0)$ satisfies the hypotheses of Theorem 1.2 so that

$$
\liminf _{t \rightarrow+\infty} \underline{u}(x, t) \geqslant \lim _{t \rightarrow+\infty} u_{n}(x, t)=c_{n},
$$

where $c_{n}=\mathbb{E}_{\rho}\left(u_{n}(x, 0)\right)$, the limit holding in all $\mathrm{L}_{\text {loc }}^{p}\left(\mathbb{R}^{N}\right)$. Hence passing to the limit as $n \rightarrow+\infty$, we obtain the result for $\underline{u}$ since $c_{n} \rightarrow \mathbb{E}_{\rho}\left(u_{0}\right)=+\infty$, thus the same holds for any other solution.

Theorems 1.4 and 1.5 follow from the conjunction of Propositions 5.1, 5.3 and 5.4.

\section{References}

[1] C. Brändle, E. Chasseigne and R. Ferreira, Unbounded solutions of the nonlocal heat equation, Commun. Pure Appl. Anal. 10 (2011), 1663-1686.

[2] E. ChASSEIGne, The Dirichlet problem for some nonlocal diffusion equations, Differential Integral Equations 20 (2007), 1389-1404.

[3] E. Chasseigne, M. Chaves and J. Rossi, Asymptotic behavior for nonlocal diffusion equations, J. Math. Pures Appl. 86 (2006), 271-291.

[4] D. EIDUS, The Cauchy problem for the nonlinear filtration equation in an inhomogeneous medium, J. Differential Equations 84 (1990), 309-318.

[5] D. EIDUs, The perturbed Laplace operator in a weighted $L^{2}$-space, J. Funct. Anal. 100 (1991), 400-410.

[6] P. FIFE, Some nonclassical trends in parabolic and parabolic-like evolutions, In: "Trends in Nonlinear Analysis", M. Kirkilionis, S. Krömker, R. Rannacher and F. Tomi (eds.). Springer, Berlin, 2003, 153-191.

[7] S. KAMIN and P. RosenaU, Nonlinear diffusion in a finite mass medium, Comm. Pure Appl. Math. 35 (1982), 113-127.

[8] S. KAMIN, Heat propagation in an inhomogeneous medium, In: "Progress in Partial Differential Equations: the Metz Surveys", 4, Pitman Res. Notes Math. Ser., Vol. 345, Longman, Harlow, 1996, 229-237.

Laboratoire de Mathématiques et Physique Théorique Universite de Tours

Parc de Grandmont

F37200 Tours, France

emmanuel.chasseigne@1mpt.univ-tours.fr

Departamento de Matemática Aplicada

Fac. de C.C. Químicas

U. Complutense de Madrid

28040 Madrid, Spain

raul_ferreira@mat.ucm.es 